\title{
Preconception omega-3 fatty acid supplementation of adult male mice with a history of developmental 2,3,7,8-tetrachlorodibenzo-p-dioxin exposure prevents preterm birth in unexposed female partners
}

\author{
Melinda E McConaha ${ }^{1}$, Tianbing Ding ${ }^{1}$, John A Lucas ${ }^{2}$, Joe A Arosh ${ }^{3}$, Kevin G Osteen ${ }^{1}$ and \\ Kaylon L Bruner-Tran ${ }^{1}$ \\ ${ }^{1}$ Women's Reproductive Health Research Center and ${ }^{2}$ Division of Reproductive Endocrinology and Infertility, \\ Department of Obstetrics and Gynecology, Vanderbilt University School of Medicine, 116121 st Avenue S, \\ MCN B-1100, Nashville, Tennessee 37232, USA and ${ }^{3}$ Department of Veterinary Integrative Biosciences, College of \\ Veterinary Medicine \& Biomedical Sciences, Texas A \& M University, College Station, Texas 77843, USA
}

Correspondence should be addressed to K L Bruner-Tran; Email: kaylon.bruner-tran@vanderbilt.edu

\begin{abstract}
We have recently reported that adult male $\mathrm{C} 57 \mathrm{BL} / 6$ mice exposed in utero to the environmental toxicant 2,3,7,8-tetrachlorodibenzop-dioxin (TCDD) confer an increased risk of preterm birth (PTB) to unexposed females. Risk of PTB was coincident with decreased placental progesterone receptor (Pgr) mRNA expression and increased toll-like receptor 4 (TIr4) mRNA expression, suggesting that toxicant exposure induced a heightened inflammatory response at the maternal-fetal interface. Since omega-3 fatty acids exhibit anti-inflammatory activity, in this study, we provided TCDD-exposed males a fish oil-enriched diet prior to mating. Although PTB was common in control females mated to TCDD-exposed males on the standard diet, fish oil supplementation of TCDD-exposed males eliminated PTB in unexposed partners. We also determined the influence of preconception, paternal fish oil supplementation on the placental inflammatory response in late pregnancy (E18.5) by examining the expression of Pgr and TIr4 mRNA as well as the expression of 15-hydroxyprostaglandin dehydrogenase (PGDH). PGDH catabolizes the inflammatory PGE2 to an inactive form; thus, reduced expression of this enzyme would promote tissue inflammation. Compared with control pregnancies, examination of E18.5 placentas arising from TCDD-exposed males on the standard diet revealed a significant increase in Tlr4 mRNA expression corresponding to a reduction in Pgr mRNA and PGDH protein expression. In contrast, fish oil supplementation of toxicant-exposed males led to normalization of placental expression of both Pgrand TIr4 mRNA and a marked increase in PGDH expression. These studies suggest that a paternal preconception diet that includes omega- 3 fatty acids prevents the toxicant-associated increase in the placental inflammatory response at late gestation, preventing PTB.

Reproduction (2011) 142 235-241
\end{abstract}

\section{Introduction}

Despite significant medical advances within the reproductive sciences, preterm birth (PTB), occurring before 37 weeks gestation, remains the leading cause of perinatal mortality and morbidity in industrialized nations (Kramer et al. 2000). Although chorioamnionitis due to bacterial or viral infection is a well-recognized cause of early human parturition (Challis et al. 2009), our understanding of other causes of PTB remains limited and the incidence of this condition continues to increase. Nevertheless, regardless of the origin, inappropriate or uncontrolled inflammation is clearly a significant contributor to the initiation of early parturition. Importantly, normal nidation is also an inflammatory event; however, inflammation is well controlled and localized to the site of embryo implantation by maternal progesterone, which acts to inhibit a generalized expression of inflammatory cytokines at the maternal-fetal interface. Although the precise mechanisms by which progesterone limits inflammatory events associated with pregnancy are not fully known, Su et al. (2009) have recently demonstrated that the immunosuppressive effects of this steroid were related to the suppression of toll-like receptor 4 (TLR4), resulting in a reduced inflammatory response. During late human pregnancy, progesterone dominance gradually subsides, eventually permitting the onset of an inflammatory cascade and parturition (Challis et al. 2009). Similarly, numerous studies in mice demonstrate that parturition in this species is also an inflammatory event, preceded by a 
disruption in progesterone action at the placentaldecidual interface (reviewed by Mendelson (2009)). Therefore, in either women or mice, impaired progesterone action prior to the end of pregnancy has been associated with PTB (Mendelson 2009).

Equally critical in regulating the timing of human and murine parturition is the synthesis and metabolism of the prostaglandins (PGs), inflammatory agents that stimulate uterine contractions and cervical ripening (Challis et al. 2002). Both PGE2 and PGF2 $\alpha$ are produced by maternal and fetal tissues during late pregnancy, with their concentrations increasing prior to and during labor (Wang \& Hirsch 2003). Within the placenta and fetal membranes, biosynthesis of these PGs is governed by prostaglandin-endoperoxide synthases 1 and 2 (PTGS1 and PTGS2; also known as cyclooxygenases 1 and 2 (COX1 and COX2)) and PGE/PGF synthases (Smith \& Song 2002). Importantly, these tissues also produce 15-hydroxyprostaglandin dehydrogenase $(\mathrm{PGDH})$, the enzyme that catabolizes PGE2 and PGF2 $\alpha$ to their inactive forms (Tai et al. 2002), thereby preventing active PGs from reaching the myometrium prior to the onset of labor. In contrast, levels of PGDH decline at the end of pregnancy, gradually permitting the transport of active PGs to the myometrium, which promotes uterine contractions and cervical ripening (Myatt \& Sun 2010). Roizen et al. (2008) demonstrated the significance of this regulatory system using mice genetically altered to produce reduced expression of $\mathrm{PGDH}$. As expected, PTB was common in pregnancies in which one or both parents were hypomorphic for PGDH expression.

Across many species, inflammation associated with infection can influence the timing of birth as a result of altered progesterone synthesis and action, resulting in inappropriate expression of PGs (Giannoulias et al. 2005, Challis et al. 2009, Bruner-Tran \& Osteen 2011); however, little is known regarding the role that the environmental toxicants may play in promoting nonmicrobial intra-uterine inflammation, which would also disrupt maintenance of pregnancy. Among the ubiquitous toxicants present within our environment is the known endocrine disruptor 2,3,7,8-tetrachlorodibenzo-p-dioxin or dioxin (TCDD), which has been linked to reproductive failure in acutely exposed women (Sharara et al. 1998). Since recent studies have suggested that early-life toxicant exposure may alter adult reproductive function (Miller et al. 2004), we established a murine model of TCDD exposure during pregnancy to examine the potential developmental effects of this toxicant on reproductive function in first generation adult offspring (toxF1 mice). Our initial study revealed that female mice exposed to TCDD in utero exhibited a dose-dependent reduction in uterine progesterone receptor (Pgr) mRNA and protein expression as adults (Nayyar et al. 2007). Not surprisingly, toxF1 female mice frequently exhibited infertility and, among mice achieving pregnancy, PTB was common (Bruner-Tran \& Osteen
2011). Additionally, PTB in toxF1 female mice was associated with a heightened sensitivity to an inflammatory challenge, a likely consequence of reduced uterine responsiveness to progesterone (Bruner-Tran \& Osteen 2011). These phenotypic changes persisted for three generations among female offspring in the absence of additional toxicant exposure. In a companion study, we found that in utero TCDD exposure of male mice conferred a similar risk of PTB following mating to an unexposed female partner (Ding et al. 2011). Thus, in utero TCDD exposure led to alterations in normal adult reproductive tract function, which negatively influences the maintenance of pregnancy, regardless of which parent was toxicant exposed. In summary, our previous developmental studies demonstrate that the toxicant-associated risk for inflammation-related PTB exists prior to conception and appear to be biologically linked to reduced placental responsiveness to progesterone. In humans and mice, the placenta is largely a paternally derived organ (Kajii \& Ohama 1977, Barton et al. 1984); therefore, a previously unrecognized therapeutic option for prevention of PTB may involve paternal intervention strategies.

In this study, we utilized our murine model to examine the potential utility of preconception paternal intervention using fish oil supplementation to prevent early parturition in female partners. Compared with females mated to TCDD-exposed males on the standard diet, we found that fish oil supplementation markedly increased pregnancy length in their unexposed partners, resulting in term delivery. Currently, women at imminent risk for PTB are most often treated with tocolytic agents; however, these anti-contraction medications cannot inhibit the inflammatory mechanisms that have already triggered the induction of labor (Simhan \& Caritis 2007). Our current findings in mice indicate that targeting the inflammatory phenotype before establishment of the maternal-fetal interface is a highly effective therapeutic strategy. Since placental-decidual communication in women also affects the timing of parturition, our studies suggest that similar preconception preventive measures may be more effective than those taken after establishment of the maternal-fetal interface.

\section{Results and Discussion}

Omega-3 fatty acids, found in fish oil, are necessary for optimal cell function but must be obtained through diet, since mice and humans have only limited ability to synthesize these compounds (Fetterman \& Zdanowicz 2009). In particular, eicosapentaenoic acid (EPA) and decosahexaenoic acid (DHA) incorporate into the plasma membrane of all cell types, displacing omega- 6 fatty acids such as arachidonic acid and reducing the availability of substrates needed for inflammatory PG synthesis (reviewed by Moreno (2009)). Recent studies have examined the efficacy of providing fish oil supplementation to women at risk for PTB (reviewed 
by McGregor et al. (2001) and Jordan (2010)); however, an increase in gestational length has not been consistently observed. Whether early-life toxicant exposure affects human risk for PTB remains unclear; nevertheless, a nutritional strategy employed prior to pregnancy may be optimal for addressing developmental defects leading to reproductive failure. Additionally, recent studies in mice demonstrating that the male partner can influence the timing of parturition further argue in favor of preconception therapy (Roizen et al. 2008, Ding et al. 2011). Data in both women and mice suggest that a placental-decidual dialogue controls the levels of progesterone receptor expression within the inflammatory microenvironment of late pregnancy, regulating the timing of parturition (Houben et al. 2009, Mendelson 2009). Since placental development in both species is markedly influenced by paternally derived genes (Kajii \& Ohama 1977, Barton et al. 1984), targeting the preconception paternal diet would likely influence placental development and potentially reduce the inflammatory responses that affect timing of birth.

In this study, we evaluated whether or not preconception fish oil supplementation of toxicant-exposed male mice (tox $1_{\text {fish }}$ ) improves pregnancy outcomes in unexposed females via a reduction in the placental response to inflammation. To this end, young adult (6-8 weeks of age) C57BL/6 male mice with and without a history of developmental TCDD exposure were transferred to a low-phytoestrogen diet with or without $5 \%$ Menhaden fish oil. Both conF1 $1_{\text {fish }}$ and toxF $1_{\text {fish }}$ males were maintained on the supplemented diet for 2-3 weeks prior to mating at which time they were transferred to the standard diet to avoid maternal fish oil supplementation. As shown in Table 1 and Fig. 1, all control female mice mated to control male (conF1) mice achieved pregnancy and delivered at term, regardless of diet. In contrast, toxicant-exposed F1 male mice maintained on the standard diet (toxF1) were frequently infertile and gestational length was significantly reduced, averaging 18.8 days $(P<0.0001$, compared with conF1 males). Toxicant-exposed males maintained on the fish oil-enriched diet (tox $F 1_{\text {fish }}$ ) exhibited a markedly improved fertility rate and gestational length in their female partners was significantly increased (19.8 days; $P<0.0001$, compared with toxF1 mice),

Table 1 Impact of paternal fish oil supplementation on 2,3,7,8tetrachlorodibenzo- $p$-dioxin-associated male infertility and preterm birth in unexposed female partners.

\begin{tabular}{lrccr}
\hline & & & \multicolumn{2}{c}{ Pregnancy outcome } \\
\cline { 4 - 5 } Mouse history & $N$ & Pregnancy & Full-term & Preterm \\
\hline ConF1 & 15 & $15 / 15(100 \%)$ & $100 \%$ & $0 \%$ \\
ConF1 $_{\text {fish }}$ & 4 & $4 / 4(100 \%)$ & $100 \%$ & $0 \%$ \\
ToxF1 $_{\text {ToxF1 }}$ & 53 & $25 / 53(47 \%)$ & $61 \%$ & $39 \%$ \\
& 11 & $9 / 11(81 \%)$ & $100 \%$ & $0 \%$ \\
\hline
\end{tabular}

which was statistically similar to control pregnancies $(P=0.1192)$. Given the striking improvement in fertility among toxF $1_{\text {fish }}$ males, following mating, a subset of mice was killed and epididymal sperm was collected. Compared with conF1 males, toxF1 mice exhibited a significant reduction in sperm density, while sperm numbers were markedly improved in toxF $1_{\text {fish }}$ males (Supplementary Table 1, see section on supplementary data given at the end of this article). These results are not entirely unexpected, since infertility in men has been linked to a reduction in spermatocyte omega-3 fatty acid content, while DHA/EPA supplementation in similar patients was associated with improved semen quality and enhanced fertility (Safarinejad 2011).

Improved fertility and longer gestational length in female partners mated to tox $\mathrm{F}_{\text {fish }}$ males clearly indicate that preconception paternal fish oil supplementation can positively influence pregnancy. Considering our current understanding of inflammation-driven term and preterm parturition, we next examined whether the beneficial effects of this dietary intervention containing fish oil protected pregnancy via a reduction in the placental response to inflammation. Since several studies suggest that a balance between Pgr and Tlr4 expression may be an important determinant in the timing of parturition (Ding et al. (2011) and references therein), we conducted quantitative RT-PCR analysis for these genes using placental tissues from mice killed in late pregnancy (E18.5). As we previously reported, placental tissues from control females mated to control males exhibited abundant Pgr mRNA and minimal T/r4 mRNA expression (Fig. 2). In contrast, placental samples from control females mated to toxF1 males exhibited a significantly higher level of TIr4 mRNA expression $(P=0.0171)$, which correlated with diminished Pgr-B/ $P g r-A B$ mRNA expression ( $P<0.025$ for both isoforms). However, Pgr expression was maintained in placental samples taken on E18.5 from control female mice mated to toxF1 $1_{\text {fish }}$ males $(P=0.4851, P g r-A B ; P=0.2984, P g r-B$; compared with conF1 mice). Among these same mice, Tlr4 mRNA expression was also reduced compared with toxF1 mice on the standard diet and was similar to observations in control animals ( $P=0.1230$; Fig. 2). Activation of TLRs has been linked to preterm parturition following bacterial infection as well as induction of normal term delivery via interaction with endogenous ligands (Patni et al. 2009). Additionally, in a mouse model, Wang \& Hirsch (2003) demonstrated that TLR4mediated induction of term and PTB was coincident with a decrease in placental expression of PGDH. Consistent with these observations, we found that late pregnancy samples obtained from females mated to toxF1 males also exhibited a dramatic decrease in PGDH immunolocalization within the fetal membranes (Fig. 3). In contrast, preconception fish oil supplementation of toxF1 male mice prevented the premature loss of $\mathrm{PGDH}$ (Fig. 3), likely contributing to the increase in 


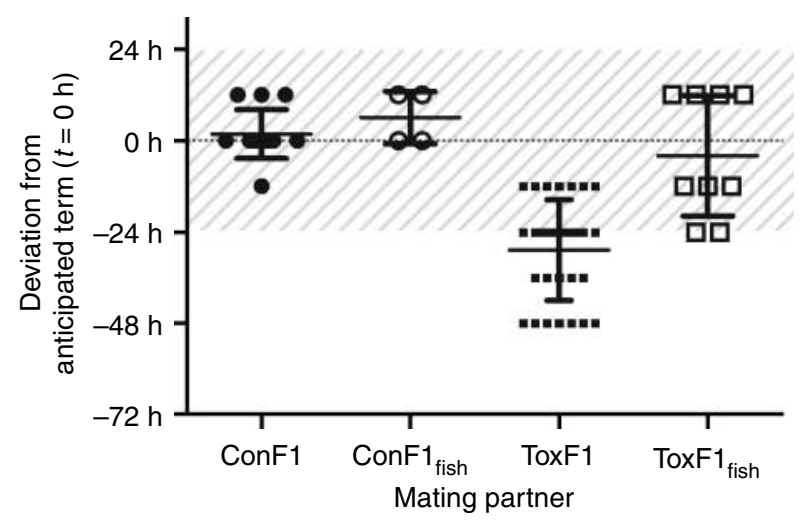

Figure 1 Effect of a fish oil-enriched diet on gestational length in control females mated to males with and without a history of in utero TCDD exposure and undergoing spontaneous labor and delivery. Anticipated time of delivery (E20) was denoted $t=0$, and actual delivery times were plotted as hours deviating from that time. Each geometric symbol represents one mouse. The inner horizontal line indicates the average hours from $t=0$ for each group of mice, while the SD is indicated by the outer lines. ConF1 female mated to conF1 male (filled circle; $N=15$ ); ConF1 female mated to $\mathrm{ConF}_{1} 1_{\text {fish }}$ male (open circle; $N=4$ ); ConF1 female mated to toxF1 male (filled square; $N=25$ ); ConF1 female mated to tox $1_{\text {fish }}$ male (open square; $N=9$ ). Shaded area represents the acceptable variation in normal delivery time $( \pm 24 \mathrm{~h})$ and which is also considered term. All males within a litter were placed on the same diet (fish oil enriched or standard diet) and multiple litters were used per group (conF1, $N=4$; $\operatorname{conF} 1_{\text {fish }}, N=2$; toxF1, $N=12$; toxF $1_{\text {fish }}, N=3$ ). $C o n F 1_{\text {fish }}$ and toxF $1_{\text {fish }}$ males were provided fish oil-supplemented diet for 2-3 weeks prior to mating.

length of gestation observed in their female partners. Taken together with our previously published findings (Bruner-Tran \& Osteen 2011, Ding et al. 2011), our current studies suggest that developmental TCDD exposure negatively impacts placental development in adulthood, resulting in alterations in inflammatory signaling at the maternal-fetal interface, which compromises pregnancy maintenance.

Despite advances in prenatal care, premature birth in industrialized countries remains a significant health care problem, perhaps suggesting that environmental exposure may contribute to this condition. We previously demonstrated that a single in utero exposure to TCDD led to reduced fertility and increased risk of PTB in three subsequent generations, strongly implicating the occurrence of epigenetic alterations within the germline of our murine model. Significantly, these data suggest that the risk of delivering preterm as an adult may be determined by the maternal or paternal fetal environment (Bruner-Tran \& Osteen (2011) and references therein). Although using animal models to unravel the potential epigenetic mechanisms of environmental toxicant action remains important, it is equally essential to translate this information into effective strategies that may reduce the negative impact of toxicants in humans. In this regard, it is currently known that the omega-3 fatty acids play an important role in regulating inflammation via multiple mechanisms, including displacement of omega- 6 fatty acids and competition for PTGS (reviewed by Calder (2003)). A recent human study has demonstrated incorporation of omega-3 fatty acids into leukocyte phospholipids and plasma within 1 week of nutritional supplementation with fish oil (Faber et al. 2011). These studies further revealed that fish oil supplementation led to displacement of omega- 6 from the cell membranes, which likely contributed to the observed modulation of in vitro immune responses. These human studies clearly support a role of dietary fish oil supplementation in rapidly modulating cell membrane composition and potentially influencing local tissue inflammation.

Although fish oil supplementation for prevention of PTB in high-risk women is beginning to be examined, intervention is typically initiated during mid-pregnancy, well after establishment of the maternal-fetal interface. Since the placenta is a key contributor in determining the timing of parturition (Houben et al. 2009, Ding et al. 2011), modulating the preconception paternal diet provides a novel and as yet unexplored strategy for the treatment of this condition in women. In contrast to most current fish oil studies, our findings suggest that dietary supplementation initiated before pregnancy and including the male partner would be more effective in preventing inflammation-related PTB.

\section{Materials and Methods}

\section{Animals}

C57BL/6 mice were purchased from Harlan Spraque-Dawley Laboratories (Indianapolis, IN, USA) and housed in the Animal Care Facility according to the National Institutes of Health

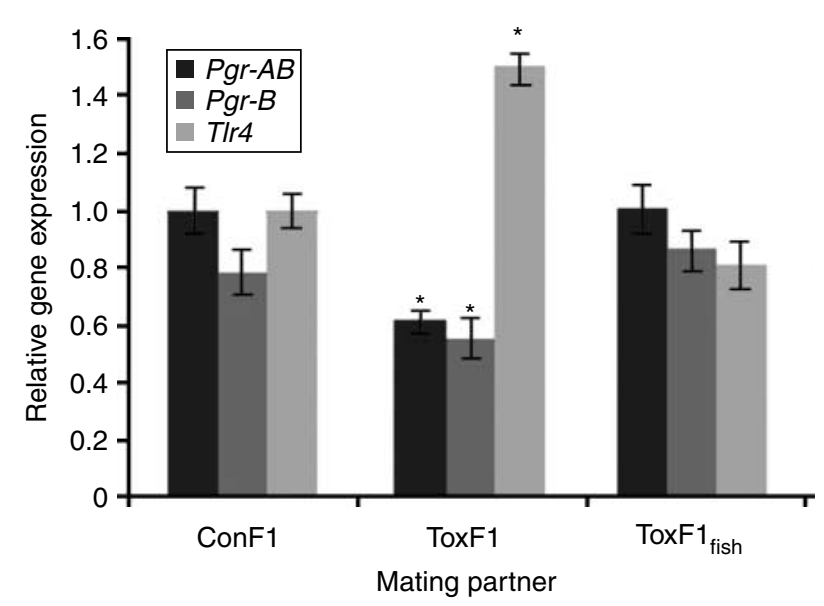

Figure 2 Expression of Pgr-AB, Pgr-B, and Tlr4 mRNA in late gestational (E18.5) placental tissues. The mRNA level of target genes was analyzed by qRT-PCR and normalized to the expression level of the housekeeping gene RplpO. mRNA is shown as fold changes compared with E18.5 control. Each bar represents the mean \pm s.D. of mRNA for multiple samples from at least three different mice per group.

Statistically significant changes $(P<0.05)$ are denoted by *. 

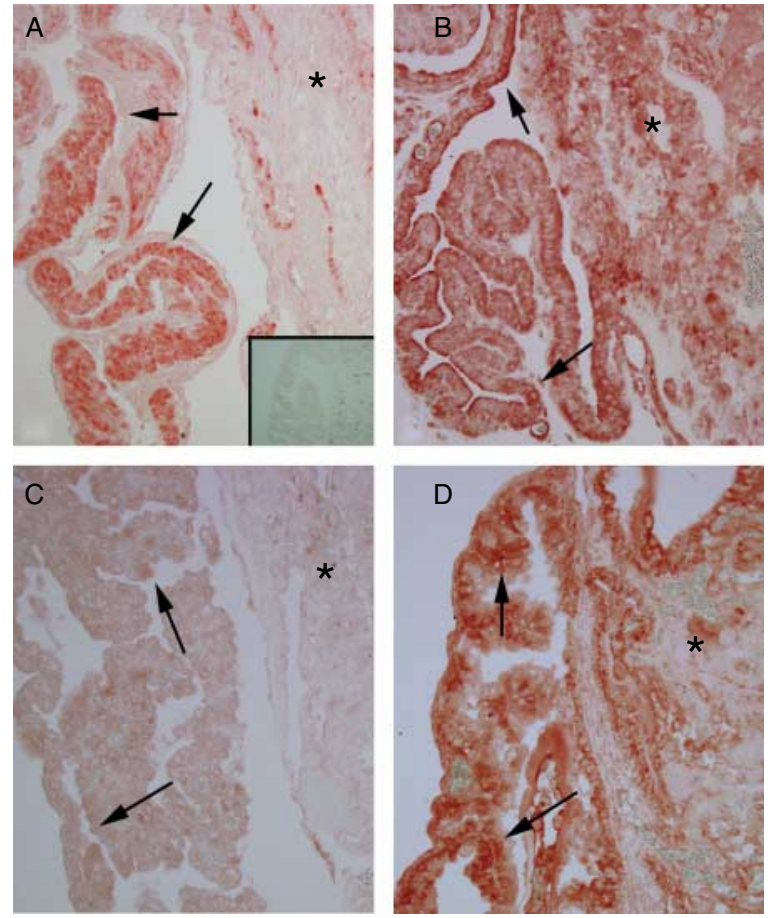

Figure 3 Immunohistochemical localization of prostaglandin dehydrogenase $(\mathrm{PGDH})$ in placental samples obtained on E18.5 from pregnant control females mated to (A) conF1 male, (B) conF1 fish male, (C) toxF1 male, or (D) toxF1 $1_{\text {fish }}$ male. $\mathrm{PGDH}$, appearing as red staining, largely localizes to the fetal membranes (arrows) and is abundant in both control samples and toxF $1_{\text {fish }}$ samples. PGDH expression is minimal in samples obtained from toxF1 mice maintained on the standard diet. Original magnification, $200 \times$. IgG control is shown as inset in (A). *Denotes placenta. Results are representative of at least four samples per group.

and Institutional Guidelines for Laboratory Animals. Animal rooms were maintained at a temperature of $22-24{ }^{\circ} \mathrm{C}$ and a relative humidity of $40-50 \%$ on a $12 \mathrm{~h}$ light: $12 \mathrm{~h}$ darkness schedule. Experiments described in this study were approved by Vanderbilt University Institutional Animal Care and Use Committee.

\section{Chemicals}

TCDD (99\%) in nonane solution was obtained from Cambridge Isotope Laboratories (Andover, MA, USA). All other chemicals were obtained from Sigma-Aldrich (St Louis, MO, USA).

\section{Rodent diets}

Purina Mills (TestDiet division; St Louis, MO, USA) provided the $5 \%$ fish oil diet, which also contained $1.5 \%$ corn oil to prevent depletion of omega- 6 fatty acids. Menhaden fish oil, generously donated by OmegaProtein (Houston, TX, USA), has an established fatty acid profile ( $\sim 40 \%$ omega- 3 fatty acids) and was processed to remove dioxins/polychlorinated biphenyls. The fish oil diet is a modification of Purina's low-phytoestrogen rodent chow, V502, which was used as the control (standard) diet. Both diets are matched for protein, total fat, and energy content. The fish oil diet was maintained in vacuum-sealed bags at $-20{ }^{\circ} \mathrm{C}$ until use and once provided to mice, replaced every 3 days.

\section{In utero $T C D D$ exposure}

Virgin C57BL/6 females ( $N=20)$, aged 10-12 weeks, were mated with intact males of similar age and examined each morning for the presence of a vaginal semen plug, denoting that copulation had occurred. The majority of normal mice will become pregnant after successful mating; thus males are removed at this time. The morning a vaginal plug is identified is considered embryonic day 0.5 (E0.5). Females were weighed prior to mating and again on E15.5 to assist with confirmation of pregnancy. Pregnant mice (F0) were exposed to TCDD $(10 \mu \mathrm{g} / \mathrm{kg})$ or vehicle corn oil by gavage at $1100 \mathrm{~h}$ Central Standard Time (CST) on E15.5. Since the half-life of TCDD in C57BL/6 mice is 11 days (Vogel et al. 2003), exposure on E15.5 results in in utero plus lactational exposure. Although this is considered a high dose of TCDD, our goal was to determine the ability of a fish oil diet to reduce the adverse affects of this compound on fertility and pregnancy; examination of the toxicological profile of TCDD was beyond the scope of this study. Importantly, the dose of TCDD used in these studies reflects the more rapid clearance of this toxicant in mice compared with humans and is well below the $\mathrm{LD}_{50}$ for adult mice of this strain $(230 \mu \mathrm{g} / \mathrm{kg}$; Vogel et al. 2003). TCDD given at this time and dose during pregnancy is not overtly teratogenic, and gestational length was not affected in the F0 animals; pups (F1 mice) were born on E20 $\pm 12 \mathrm{~h}$.

\section{Mating scheme of F1 mice and monitoring of pregnancy}

A single female was placed with a single male and observed each morning for a vaginal plug. Males that produced three positive vaginal plugs, but no observable pregnancy, were considered infertile. Following identification of vaginal plug (E0.5), males were removed and parturition expected on E20. Preterm parturition in mice has been defined as spontaneous labor $\geq 24 \mathrm{~h}$ prior to term (Roizen et al. 2008, Ding et al. 2011). After E17, all mice were monitored twice daily for the timing of delivery of the first pup.

\section{Killing of pregnant females and collection of tissues}

Spontaneous PTB in toxicant-exposed animals typically occurs on or before E18.5 (Bruner-Tran \& Osteen 2011, Ding et al. 2011); therefore, in this study, a subset of pregnant females from all groups were killed at 1400-1500 h CST on E18.5 and tissues were collected as described previously (Ding et al. 2011). Briefly, pregnant females were weighed immediately prior to killing by cervical dislocation under anesthesia. The uterus and cervix were removed in toto, and placentas were dissected from the decidua. Half of all placentas were flash frozen for RNA extraction and stored at $-80{ }^{\circ} \mathrm{C}$, and the remainder was formalin fixed. All cervices were formalin fixed. Since only a subset of females mated to toxF1 males can be expected to deliver preterm, cervices were histologically examined for evidence of cervical ripening (mucin via PAS staining and 
collagen degradation via Masson's trichrome staining, data not shown) to aid in selection of appropriate tissues for RT-PCR analysis. No pregnancies resulting from mating with conF1 or toxF $1_{\text {fish }}$ males exhibited extensive cervical ripening.

\section{Histochemistry/immunohistochemistry}

Fixed samples were processed, paraffin embedded, and sectioned at $5 \mu \mathrm{m}$ at the Vanderbilt Histology Core Laboratory. Cervices were subjected to hematoxylin and eosin (H\&E), PAS, and Masson's trichrome staining by standard methodology.

Immunohistochemical localization of PGDH protein in placenta and fetal tissues was performed using a Vectastain Elite ABC Kit (Vector Laboratories, Inc., Burlingame, CA, USA) according to the manufacturer's protocol. Endogenous peroxidase activity was removed $(0.3 \%$ hydrogen peroxide/methanol), and sections were blocked in $10 \%$ goat serum for $1 \mathrm{~h}$ at room temperature. Slides were incubated with the anti-human rabbit polyclonal $\mathrm{PGDH}$ antibody (1:500, Cayman Chemicals, Ann Arbor, MI, USA) overnight at $4{ }^{\circ} \mathrm{C}$ followed by goat anti-rabbit IgG biotinylated secondary antibody for $45 \mathrm{~min}$ at room temperature. Rabbit serum was used as the negative control (1:500). Slides were viewed with an Olympus BX51 microscope system and images were captured using an Olympus DP71 digital camera (Olympus, Center Valley, PA, USA).

\section{Quantitative RT-PCR analysis}

Total RNA was isolated from frozen placental tissues with TRIzol (Invitrogen) and purified using the RNeasy Mini Kit (Qiagen). cDNA from $1 \mu \mathrm{g}$ of total RNA was synthesized using the iScript cDNA Synthesis Kit (Bio-Rad) and random decamer primers. Reactions were performed in duplicate in a Bio-Rad CFX96 Real-time thermocycler system. The ribosomal protein, large, P0 (RplpO) gene was used as an endogenous control. Results were evaluated using the $\Delta \Delta C_{\mathrm{t}}$ method, where delta was calculated as (target $\left.C_{\mathrm{t}}\right)-\left(\right.$ RplpO $\left.C_{\mathrm{t}}\right)$, and the relative quantity of target gene expression was calculated by the $\Delta \Delta C_{t}$ as $2^{\left.-\left(\text {(experiment sample } \Delta C_{\mathrm{t}}\right)-\left(\text { control sample } \Delta C_{\mathrm{t}}\right)\right)}$. Primers (forward and reserve) and the thermal cycling protocol using the CFX96 Realtime System have been previously described (Ding et al. 2011).

\section{Killing of males and analysis of sperm density}

Following a minimum of $72 \mathrm{~h}$ after mating, adult (12-16 weeks) males were killed by cervical dislocation under anesthesia; sperm was collected from epididymal cauda, and density was determined by standard methodology (Wang 2002). For each sample, two counts were taken and density was calculated using the following equation: sperm density $=$ (mean count $\times$ dilution factor)/cauda weight (mg).

\section{Statistical analysis}

Statistical analysis was performed using GraphPad Prism (La Jolla, CA, USA). All data are expressed as mean \pm s.D. Statistical comparisons between two experimental groups were determined using the parametric Student's $t$-test (for normally distributed populations). A $P$ value of $<0.05$ was considered statistically significant.

\section{Supplementary data}

This is linked to the online version of the paper at http://dx.doi. org/10.1530/REP-11-0070.

\section{Declaration of interest}

The authors declare that there is no conflict of interest that could be perceived as prejudicing the impartiality of the research reported.

\section{Funding}

Supported by NCCAM R21AT006245 (K B T), NIEHS R01ES14942 (K G O), Vanderbilt University Medical Center (K B T), and the International Endometriosis Association (K G O).

\section{Acknowledgements}

We gratefully acknowledge the donation of highly purified Menhaden fish oil by OmegaProtein (Houston, TX, USA) and to Dr Carrie Schultz (Purina TestDiets) for expert assistance in formulating the fish oil diet. We also acknowledge the assistance of the Vanderbilt Histology Core Laboratory.

\section{References}

Barton SC, Surani MA \& Norris ML 1984 Role of paternal and maternal genomes in mouse development. Nature 311 374-376. (doi:10.1038/ 311374a0)

Bruner-Tran KL \& Osteen KG 2011 Developmental exposure to TCDD reduces fertility and negatively affects pregnancy outcomes across multiple generations. Reproductive Toxicology 31 351-358. (doi:10. 1016/j.reprotox.2010.10.003)

Calder PC 2003 N-3 polyunsaturated fatty acids and inflammation: from molecular biology to the clinic. Lipids 38 343-352. (doi:10.1007/ s11745-003-1068-y)

Challis JR, Sloboda DM, Alfaidy N, Lye SJ, Gibb W, Patel FA, Whittle WL \& Newnham JP 2002 Prostaglandins and mechanisms of preterm birth. Reproduction 124 1-17. (doi:10.1530/rep.0.1240001)

Challis JR, Lockwood CJ, Myatt L, Norman JE, Strauss JF III \& Petraglia F 2009 Inflammation and pregnancy. Reproductive Sciences 16 206-215. (doi:10.1177/1933719108329095)

Ding T, McConaha M, Boyd KL, Osteen KG \& Bruner-Tran KL 2011 Developmental dioxin exposure of either parent is associated with an increased risk of preterm birth in adult mice. Reproductive Toxicology $\mathbf{3 1}$ 344-350. (doi:10.1016/j.reprotox.2010.11.003)

Faber J, Berkhout M, Vos AP, Sijben JW, Calder PC, Garssen J \& van Helvoort A 2011 Supplementation with a fish oil-enriched, high-protein medical food leads to rapid incorporation of EPA into white blood cells and modulates immune responses within one week in healthy men and women. Journal of Nutrition 141 964-970. (doi:10.3945/jn.110.132985)

Fetterman JW Jr \& Zdanowicz MM 2009 Therapeutic potential of n-3 polyunsaturated fatty acids in disease. American Journal of HealthSystem Pharmacy 66 1169-1179. (doi:10.2146/ajhp080411)

Giannoulias D, Haluska GJ, Gravett MG, Sadowsky DW, Challis JR \& Novy MJ 2005 Localization of prostaglandin $\mathrm{H}$ synthase, prostaglandin dehydrogenase, corticotropin releasing hormone and glucocorticoid receptor in rhesus monkey fetal membranes with labor and in the presence of infection. Placenta 26 289-297. (doi:10.1016/j.placenta. 2004.07.005)

Houben ML, Nikkels PG, van Bleek GM, Visser GH, Rovers MM, Kessel H, de Waal WJ, Schuijff L, Evers A, Kimpen JL et al. 2009 The association 
between intrauterine inflammation and spontaneous vaginal delivery at term: a cross-sectional study. PLoS ONE 4 e6572. (doi:10.1371/journal. pone.0006572)

Jordan RG 2010 Prenatal omega-3 fatty acids: review and recommendations. Journal of Midwifery \& Women's Health 55 520-528. (doi:10. 1016/j.jmwh.2010.02.018)

Kajii T \& Ohama K 1977 Androgenetic origin of hydatidiform mole. Nature 268 633-634. (doi:10.1038/268633a0)

Kramer MS, Demissie K, Yang H, Platt RW, Sauve R \& Liston R 2000 The contribution of mild and moderate preterm birth to infant mortality. Fetal and Infant Health Study Group of the Canadian Perinatal Surveillance System. Journal of the American Medical Association 284 843-849. (doi:10.1001/jama.284.7.843)

McGregor JA, Allen KG, Harris MA, Reece M, Wheeler M, French JI \& Morrison J 2001 The omega-3 story: nutritional prevention of preterm birth and other adverse pregnancy outcomes. Obstetrical \& Gynecological Survey 56 (5 Supplement 1) S1-S13. (doi:10.1097/ 00006254-200105001-00001)

Mendelson CR 2009 Minireview: fetal-maternal hormonal signaling in pregnancy and labor. Molecular Endocrinology 23 947-954. (doi:10. 1210/me.2009-0016)

Miller KP, Borgeest C, Greenfeld C, Tomic D \& Flaws JA 2004 In utero effects of chemicals on reproductive tissues in females. Toxicology and Applied Pharmacology 198 111-131. (doi:10.1016/j.taap.2003.07.016)

Moreno JJ 2009 Differential effects of arachidonic and eicosapentaenoic acid-derived eicosanoids on polymorphonuclear transmigration across endothelial cell cultures. Journal of Pharmacology and Experimental Therapeutics 331 1111-1117. (doi:10.1124/jpet.109.157891)

Myatt L \& Sun K 2010 Role of fetal membranes in signaling of fetal maturation and parturition. International Journal of Developmental Biology 54 545-553. (doi:10.1387/ijdb.082771/m)

Nayyar T, Bruner-Tran KL, Piestrzeniewicz-Ulanska D \& Osteen KG 2007 Developmental exposure of mice to TCDD elicits a similar uterine phenotype in adult animals as observed in women with endometriosis. Reproductive Toxicology 23 326-336. (doi:10.1016/j. reprotox.2006.09.007)

Patni S, Wynen LP, Seager AL, Morgan G, White JO \& Thornton CA 2009 Expression and activity of toll-like receptors 1-9 in the human term placenta and changes associated with labor at term. Biology of Reproduction 80 243-248. (doi:10.1095/biolreprod.108.069252)
Roizen JD, Asada M, Tong M, Tai HH \& Muglia LJ 2008 Preterm birth without progesterone withdrawal in 15-hydroxyprostaglandin dehydrogenase hypomorphic mice. Molecular Endocrinology 22 105-112. (doi:10.1210/me.2007-0178)

Safarinejad MR 2011 Effect of omega-3 polyunsaturated fatty acid supplementation on semen profile and enzymatic anti-oxidant capacity of seminal plasma in infertile men with idiopathic oligoasthenoteratospermia: a double-blind, placebo-controlled, randomised study. Andrologia 43 38-47. (doi:10.1111/j.1439-0272.2009.01013.x)

Sharara FI, Seifer DB \& Flaws JA 1998 Environmental toxicants and female reproduction. Fertility and Sterility 70 613-622. (doi:10.1016/S00150282(98)00253-2)

Simhan HN \& Caritis SN 2007 Prevention of preterm delivery. New England Journal of Medicine 357 477-487. (doi:10.1056/NEJMra050435)

Smith WL \& Song I 2002 The enzymology of prostaglandin endoperoxide $\mathrm{H}$ synthases-1 and -2. Prostaglandins \& Other Lipid Mediators 68-69 115-128. (doi:10.1016/S0090-6980(02)00025-4)

Su L, Sun Y, Ma F, Lü P, Huang H \& Zhou J 2009 Progesterone inhibits tolllike receptor 4-mediated innate immune response in macrophages by suppressing NF-kappaB activation and enhancing SOCS1 expression. Immunology Letters 125 151-155. (doi:10.1016/j.imlet.2009.07.003)

Tai HH, Ensor CM, Tong M, Zhou H \& Yan F 2002 Prostaglandin catabolizing enzymes. Prostaglandins \& Other Lipid Mediators 68-69 483-493. (doi:10.1016/S0090-6980(02)00050-3)

Vogel CF, Zhao Y, Wong P, Young NF \& Matsumura F 2003 The use of C-src knockout mice for the identification of the main toxic signaling pathway of TCDD to induce wasting syndrome. Journal of Biochemical and Molecular Toxicology 17 305-315. (doi:10.1002/jbt.10096)

Wang Y 2002 Epididymal sperm counts. In Current Protocols in Toxicology, pp 16.6.1-16.6.5. Ed. G Coruzzi. Hoboken, New Jersey, USA: John Wiley \& Sons, Inc.

Wang H \& Hirsch E 2003 Bacterially-induced preterm labor and regulation of prostaglandin-metabolizing enzyme expression in mice: the role of toll-like receptor 4. Biology of Reproduction 69 1957-1963. (doi:10. 1095/biolreprod.103.019620)

Received 8 March 2011

First decision 26 April 2011

Accepted 8 June 2011 